\title{
Psychiatry and the neurosciences
}

The last 10 years have witnessed encouraging developments in our incipient understanding of some of the brain events that form the material substratum for normal and abnormal psychic function and behaviour. Notable examples are the discovery of the role of dopamine in Parkinsonism and the role of serotonin and noradrenaline in manic-depressive disorders. This has led, for the first time in neuropsychiatry, to the design of a treatment procedure (L-dopa) on rational grounds, and to plausible explanations for the mechanism of action of agents precipitating depression-for example, reserpine, and alleviating it-for example, the tricyclic drugs. Of course, many gaps need to be filled in and there is still dispute over details, but most workers in this field would agree that the 'amine hypothesis' of the affective disorders is in a healthy condition. The picture in schizophrenia is much less pronising, but at least some research work is under way centring on the only three facts that we know about the pathophysiology of the illness-namely, the abnormal susceptibility of some schizophrenics to methionine, the histamine resistance found in many cases, and the antiadrenergic and anti-dopaminergic action of many drugs-for example, the phenothiazines and pimozide-effective in the schizophrenias.

We are furthermore gaining a little understanding of the role that different neurotransmitters may play in the brain in functional terms. There is now evidence to support Kety's hypothesis (1972) that noradrenaline (NA) may be the chemical signal to the neurones in the brain 'positive reinforcement received-now print'. NA seems also to be concerned in the control of sleep and feeding behaviour. Dopamine is concerned with the initiation of all movement as well as other aspects of motor function. Serotonin (5-HT) seems to be concerned with basic mood as well as perception, sleep, and various meta-organizational systems. Acetylcholine is involved in the reticular activating system and excessive $\mathrm{ACh}$ release may signal negative reinforcement relayed by the 'pain mechanism'. The transmission of basic information about the external and internal environment may depend on glutamate (excitatory) and GABA (inhibitory) as neurotransmitters.

These biochemical facts and theories enable us to put some order into the clinical facts. For example, if NA is concerned in the regulation of positive reinforcement, sleep, and eating behaviour, it becomes understandable that, if effective levels of NA in the brain decline in depression, then absence of enjoyment, and disturbances of sleep and appetite result. However, it is, of course, possible that some psychiatric syndromes may depend on biochemical mechanisms in the brain as yet hardly explored. For example,

1. The role of histamine in brain function is quite unknown. Antihistamines have well-known central effects-drowsiness, for example-and schizophrenics are known to be abnormally histamine resistant.

2. The bulk of our present knowledge of brain function centres on synaptic mechanisms. Our current picture of the brain in action differs little from the 'telephone exchange' and 'nerve net' models of earlier workers. In these models learning was thought to be due to small changes in synaptic knobs produced by repeated activity. There is gathering evidence that protein synthesis (and hence the DNA-RNA system) is concerned in learning and sleep. An alternative hypothesis has recently been put forward (Smythies, 1969) that some transmitters have more to do than simply to trigger the depolarization (excitation) or hyperpolarization (inhibition) of the postsynaptic cell membrane. Information specific transmitters-for example, NA, 5-HT-or their metabolites, may be taken up into the post-synaptic cell and there modulate cell functions such as protein synthesis and axoplasmic flow. The metabolic product of serotonin is 5-HIAA which is very close to the plant auxin IAA in structure. The latter is known to effect its role on growth stimulation by binding to 
specific proteins in the nucleus and modulating DNA directed protein synthesis. Jouvet (1968) has suggested that metabolites of serotonin and NA may be concerned in the regulation of sleep.

The role of catecholamine-O-methyl transferase (COMT) in the adrenergic synapse has always been somewhat mysterious. The bulk of NA released is taken back unaltered into the presynaptic terminal and only a small percentage is metabolized by COMT which seems to be located in or near the post-synaptic membrane. Thus the role of COMT is clearly not analagous to that of AChE in the cholinergic terminal. It is possible that its function in making metanoradrenaline is to make not a mere inactive end-product, but something which may be taken up into the post-synaptic cell, there to act as a signal of the degree of NA release in the cellular environment (and hence the desirability or otherwise of continuing the present activity of the cell). Mescaline, and a number of its hallucinogenic relatives (which are chemically related to meta-dopamine), have recently been shown to be potent inhibitors of axoplasmic transport (Paulson and McClure, 1973). Furthermore, there is a close correlation between the hallucinogenic potency of a compound and its ability to block axoplasmic transport. Clearly, this offers a second means of control of the activity of the postsynaptic by the presynaptic cell. Recently it has been shown (Carnegie et al., 1972) that serotonin and certain of its hallucinogenic derivatives will block the reaction between the basic brain protein of myelin and antibodies specific for this protein which may also have some functional significance.

Thus possibilities open up that the basic biochemical lesion in schizophrenia and other psychoses may lie in the genetic mechanism in strategic neurones and/or in mechanisms controlling axoplasmic flow as well as in the more fashionable locus of the synapse.

This hypothesis suggests that the processes of learning, and hence the aberrations of learning that make up the neuroses, may involve not so much small structural changes in presynaptic knobs but possibly much more extensive chemical changes in the post-synaptic neurone. The new proteins formed in the post-synaptic cell as the result of the learning process could modify its receptors both quantitatively or qualitatively or modulate its own synaptic or other functions. Hence quite new nerve nets could be set up, since connections between neurones are chemospecific. The nervous system may be much more dynamic in its wiring patterns than the older 'nerve net' models suggested.

Neurochemistry has made progress in areas other than the major psychoses. For example, the basic mechanisms of drug addiction have recently been subject to an intensive investigation. The picture that emerges is that drugs of addiction interfere with the basic chemical mechanisms of reinforcement. They can do this either by switching on the mechanism underlying positive reinforcement (as do amphetamine and cocaine) or by switching off the mechanism underlying negative reinforcement (as does morphine). In either case the chemical itself takes over the role of the major reinforcer of behaviour. A secondary effect is that enzyme synthesis is induced in the brain in an attempt to overcome the drug-induced abnormality. This leads, when the drug is stopped, to the syndrome of drug withdrawal by overactivity of the newly synthesized enzymes.

So far I have been discussing events largely at the chemical and cellular level. However, progress has also been made at the levels of those brain mechanisms of interest to psychiatrists. In the face of great experimental difficulties of execution and interpretation, we are developing some sketchy preliminary ideas of the role of different nuclei and areas of the brain in higher nervous functionsfor example: the role of different parts of the limbic system in the control of learning, motivation, and emotion; the different function of the two cerebral hemispheres as revealed by split-brain preparations (human and animal) (Smythies, 1970). New concepts of global brain function have been provided by the models of holography and holophony. However, there is less agreement whether the application of this new knowledge in the field of psychosurgery has been so effective.

Lastly, I should like to comment on some more basic philosophical issues. It might appear, from what I have said so far, that I retain an uncritical admiration for neuroscientists and all their works. However, neuroscience is no more free than any other branch of science from the problem of pollution-that is, the unnoticed generation of undesirable side-effects from one's scientific work. Atom bombs, DDT, and over-population are familiar sources of distress to physicists, chemists, 
and doctors. But psychiatrists, psychologists, and neuroscientists are not immune from this melancholy process. Some note has been taken of late of the scandals of Soviet "political' psychiatry and complaints have been made in some quarters concerning the activity of some over-enthusiastic psychosurgeons. Some renegade psychiatrists have even claimed that the bulk of psychiatry itself is an exercise in mind-pollution and alienation.

More serious, in my opinion, has been the idea widely held by many scientists and clinicians in these fields, that a monistic theory of mind--brain relation has somehow been established, by advances in neuroscience, neuropsychiatry, and psychology, as an unshakeable scientific fact. This is largely due to the widespread ignorance among these scientists of the philosophical issues involved. Our ever-increasing knowledge of how the brain works is quite irrelevant to the quite separate problem of what is the relation between mental events and brain events. Our clinical knowledge of the obvious relationship between states of mind and consciousness and the state of the brain does not carry with it the answer to the mind-brain problem either. These facts are quite neutral between the two opposing hypotheses-psychoneural identity and psychoneural interaction. No one doubts that mental events are determined by brain events. However, a set of events $a$ can be determined by a set of events $b$ either because (1) $a=b$ or (2) because $a$ and $b$ are causally connected. I have argued elsewhere (Smythies, 1956) that the theory of psychoneural identity has a number of serious defects and thus the matter can by no means be regarded as decided. Since matters of great religious and social importance depend on our attitude to this problem, one may hope that the excessive dogmatism that has clouded this field for a century may give way to a more modest and open-minded attitude. The dogmatic statement that men are only machines, it can be argued, leads other lesssophisticated and more evil men to tend to treat their fellow humans as (expendable) machinesat least more than they would have done if they had not abandoned the alternative Greco-JudeoChristian view of man, in favour of the new scientific dogma; hence Belsen, etc.

Scientists are as likely to suffer from attacks of hubris as anyone else. At the end of the 19th century physicists passed through an era of complacency where it was thought every problem in physics was solved, or on the point of being solved. As Arthur Koestler has often pointed out, physicists do not subscribe to this doctrine today. The hubris virus has passed on to infect many neuroscientists, psychologists and even, it grieves one to say, some philosophers, for example those of the Australian School. However, one can be certain that all reductionist attempts to explain 'mind' in terms of brain chemistry (only), or reinforcement schedules (only), or computer logic (only), or on any combinations of these, will fail (Koestler and Smythies, 1969). The facts of conscious experience are irreducible and must enter in their own right as basic irreducible elements, into any comprehensive account of mind (Beloff, 1962; Eccles, 1971).

J. R. SMYTHIES

\section{REFERENCES}

Belofi, J. (1962). The Existence of Mint. MacGibbon and Kee: London.

Carnegie, P. R., Smythies, J. R., Caspary, E. A., and Field, E. J. (1972). Interaction of hallucinogenic drugs. Nature, $240,561-563$.

Eccles, J. C. (1971). Facing Reality. Longmans: London.

Jouvet, L. (1968). Neurophysiology of the states of sleep. In The Neurosciences, pp. 529-544. Edited by G. C. Quarton, T. Melnechnk, and F. O. Schmitt. Rockefeller Press: New York.

Kety, S. S. (1972). Brain catecholamines, affective states and memory. In The Chemistry of Mood, Motivation, and Memory, pp. 65-80. Edited by J. L. McGaugh. Plenum: New York.
Koestler, A., and Smythies, J. R. (1969) (eds). Beyond Reductionism. Hutchinson: London.

Paulson, J. C., and McClure, W. O. (1973). Inhibition of axoplasmic transport by mescaline and other trimethoxyphenylalkylamines. Molecular Pharmacology, 9, 41-50.

Smythies, J. R. (1965). Analysis of Perception. Routledge: London.

Smythies, J. R. (1969). Neurochemical aspects of information processing in the brain. Communications in Behavioral Biology, Part A, 3, 24I-248.

Smythies, J. R. (1970). Brain Mechanisms and Behaviour. 2nd edn. Blackwell:"Oxford. 